\title{
Motherhood and well-being in young breast cancer survivors
}

Cynthia Wan*,1, Isabelle Arès², Alexandre Gareau¹, Katherine A Collins ${ }^{3}$, Sophie Lebel $^{1}$ \& Catherine Bielajew ${ }^{1}$

${ }^{1}$ School of Psychology, University of Ottawa, 136 Jean Jacques Lussier Pvt, Ottawa, ON K1N 6N5, Canada

${ }^{2}$ The Royal Ottawa Mental Health Centre, 1145 Carling Ave, Ottawa, ON K1Z 7K4, Canada

${ }^{3}$ Department of Psychology, Concordia University of Edmonton, 7128 Ada Blvd NW, Edmonton, AB T5B 4E4, Canada

*Author for correspondence: cwan062@uottawa.ca

\section{Practice points}

- 15 elements of well-being were identified in the literature and exploratory factor analysis revealed a four-factor structure of well-being in breast cancer survivors (BCS): recurrence worries, physical health, psychological adjustment and illness intrusiveness.

- Analyses revealed that there was a stronger relationship between psychological adjustment and illness intrusiveness for young BCS with children.

- Psychological distress (i.e., lack of psychological adjustment), illness intrusiveness and fear of recurrence co-occurred more frequently in young BCS with children than in BCS without children, thus potentially compromising their well-being.

- Our findings indicate that young BCS with children are most in need of screening and interventions in these areas, and such initiatives should be incorporated in their medical care and visits.

Aim: This exploratory study aimed to examine differences in well-being between young breast cancer survivors (BCS) with and without children. Materials \& methods: Participants $(n=816)$ completed an online survey relating to quality of life, illness intrusiveness, fear of cancer recurrence, stress and social support. Results: Exploratory and confirmatory factor analyses revealed similar models of well-being between both groups, but with a stronger relationship between psychological adjustment and illness intrusiveness for BCS with children ( $r=-0.779,95 \% \mathrm{Cl}:-0.711,-0.848$ vs $r=-0.525,95 \% \mathrm{Cl}:-0.423,-0.627)$. Conclusion: Parenting compromises the overall well-being of young BCS with children and they would therefore benefit from interventions and social and oncological support programs, especially for those caring for minor children.

First draft submitted: 30 September 2017; Accepted for publication: 11 January 2018; Published online: 23 January 2018

Keywords: breast cancer survivorship • children • distress $\bullet$ fear of cancer recurrence $\bullet$ illness intrusiveness $\bullet$ quality of life

In a recent population-based study of cancer survivors, Weaver et al. [1] examined the prevalence of parental cancer via the United States National Health Interview Survey (2000-2007); they identified 13,385 adults with a history of cancer with one or more minor children under the age of 18 . Only adults with minor children who were diagnosed with cancer less than 2 years before the survey were included in the study $(\mathrm{n}=1769)$, but of that subset, approximately $85 \%$ of them were diagnosed under the age of 45 [1]. In the USA, the average age of breast cancer diagnosis is 61 years for women; but $11 \%$ of all new cases occur in women under the age of 45 [2]. Following these guidelines, we operationalized 'young breast cancer survivors' as individuals with a diagnosis of cancer under the age of 45 and who were currently considered cancer free [2]. A diagnosis of breast cancer at a young age can interfere with the demands of early adulthood and the short- and long-term effects of treatment can be especially problematic for this group [3]. Young breast cancer survivors with children (BCS with children) may, therefore, be a particularly vulnerable group because the stressors that accompany motherhood may compound the difficulties experienced in their survivorship trajectory [4]. Despite the need to tailor interventions and survivorship care to this particular group, the literature on this subject remains scant [5].

Future Medicine 


\section{Breast cancer \& motherhood}

BCS with children tend to sacrifice their own need for care and rely heavily on social support in an effort to meet their children's needs [6,7]. Ultimately, they describe a sense of loss in the quality time spent with their children, feel guilty for being unavailable [7], and they display worries regarding their children's well-being in the event that they should die $[4,8]$. Not surprisingly, this group displays high levels of psychological distress [9]; and maternal distress in BCS has important consequences on marital relationships, family functioning, and the ability of children to cope with the situation $[9,10]$. However, the current existing studies on parental cancer and motherhood are typically qualitative in nature, assessing one aspect of well-being, or providing a systematic review of the limited literature. To our knowledge, there are no published studies to date examining a model of well-being in young BCS with and without dependent children.

\section{Well-being in young BCS}

We performed a literature review and identified elements of short- and long-term well-being that are salient to all young BCS (e.g., health-related quality of life, fear of recurrence and subjective levels of stress), while trying to capture unique aspect of well-being that can be associated with being a BCS with children (e.g., availability of social support, extent of illness intrusiveness). Based on this review, we designed a study to examine those elements of well-being between BCS with and without children. Specifically, we examined the following elements: healthrelated quality of life, fear of recurrence (including worries pertaining to health, death, womanhood), the extent of intrusiveness that breast cancer has on various aspects of their life, amount of perceived stress they experience day-to-day and the availability of social support.

It has been reported in the literature that young breast cancer patients tend to have a worse 5-year survival rate compared with that of older breast cancer patients, and they are often diagnosed with more advanced stage disease and experience more aggressive tumor characteristics [11]. In fact, diagnosis at a young age was reported as a risk factor for mortality [11]. It is, therefore, not a surprise that young BCS often report disturbances in their short- and long-term health-related quality of life, as measured by physical and social functioning, degree of bodily pain experienced, vitality and quality of mental health [12]. Preliminary evidence indicates that BCS with children report lower health-related quality of life than those without children [12]. However, Bloom et al.'s results [12] must be interpreted with caution because it is not likely representative of all young BCS. Specifically, their results are based on a group of women who did not experience a breast cancer recurrence or metastasis and they lacked a comparison group, which may affect the conclusions that they have drawn. Despite the limitations, it is likely that BCS with children exhibit lower health-related quality of life. Other studies have suggested that BCS with children may experience reduced health-related quality of life due to elevated levels of distress and physical exhaustion due to their added role of parenting in addition to coping with recovery $[8,9]$.

In relation to health-related quality of life and of greatest concern among BCS due to its pervasive nature, is the fear of recurrence [13]. Fear of recurrence as defined in the current study is measured by assessing fears that cancer recurrence would affect one's physical and emotional health, role and responsibilities at work and at home, femininity and sexuality, possibility of death as well as overall fear (frequency, potential for upset, intensity) [14]. Various studies have suggested that while most, if not all, BCS harbor worries and fears toward cancer recurrence, BCS with children report greater fears of cancer progression and cancer recurrence than BCS without children [1516]. Moreover, it has been reported that the sequence of childbirth and breast cancer may play a role in breast cancer recurrence. For example, there is a higher likelihood of breast cancer recurrence and metastasis for women who were diagnosed with breast cancer within 5 years of childbirth [17]; however, pregnancies during and/or after breast cancer treatment do not appear to increase the risk of recurrence [18].

Although there are obvious concerns and fears toward cancer recurrence, another toll is the interference and disruption that it will cause in the survivor's life (again). Once diagnosed with a chronic illness, due to the nature of the illness and its accompanying treatment and aftercare, there will be inevitably various domains in the individual's life that will be disrupted, this is termed as illness intrusiveness. In particular, young BCS report that their illness interferes with the demands of early adulthood [3], and for those caring for children, the physical impact of cancer, such as pain, fatigue and general malaise are especially disruptive to their ability to parent [8]. In addition to being mindful of one's health-related quality of life and taking her degree of fear of recurrence in consideration, one's amount of perceived stress in their day-to-day life also plays a major role in their well-being [19]. Broadly, stress can be classified as negative, positive or neutral, and its impact on one's well-being are highly dependent on the individual's appraisal of the situation. In the context of young BCS, studies have reported that parenting and its 
associated responsibilities often pose an added burden to the already heavy demands of their illness and young BCS with children often display higher levels of perceived stress [5,6].

Although we have highlighted a few salient elements toward the assessment of well-being, some of which will likely to negatively affect one's well-being, we identified a positive correlate: the availability of social support. Emotional and practical support from family, friends and health providers is essential to the well-being of BCS [20]. Studies show that young BCS experience a decline in their social support over the course of their illness [12,21], many of whom feel unsupported by their peers. Young BCS with children, in particular, rely heavily on others to help manage their parenting responsibilities [8], and many have reported unmet needs for support from health providers with matters related to parenting [5]. Difficulties accessing this support may therefore place this group at risk for poor adjustment [22].

\section{Current study: aim \& hypotheses}

Given the prevalence of parental cancer, it is alarming that very few studies have investigated differences in wellbeing in cancer survivors with and without children $[5,23]$. The lack of studies comparing these two groups in the literature prevents a clear understanding of the well-being of BCS with children and hinders the advancement of clinical and post-treatment care. Our aim was to investigate whether having children affects the well-being of young BCS. We acknowledge that many factors contribute to one's well-being, but we will only focus on the following five correlates: health-related quality of life, fear of recurrence, illness intrusiveness, perceived stress and availability of social support. We used factor analytic techniques to explore the grouping of these elements and compared these elements between young BCS with and without children. We achieved this by first exploring the factorial structure of the preidentified elements of well-being with a sample of young BCS with children, and testing the invariance of that structure in a new sample of young BCS with and without children.

While we expected young BCS with and without children to share the same factorial structure of well-being, it is possible that differences in factor covariances and factor means could be found. We hypothesized young BCS with children to experience greater physical demands due to the responsibility of caring for children, thus we expect to see a stronger relationship between physical well-being (e.g., health-related quality of life, vitality, bodily pain, physical and social functioning) with illness intrusiveness and their psychological well-being (e.g., degree of perceived stress, mental health). Finally, we expected fear of cancer recurrence to be more closely related to psychological well-being in BCS with children given the potential consequences a recurrence could have on their families.

\section{Materials \& methods Participants}

In this study, young BCS were defined as women who were diagnosed with breast cancer at the age of 45 or younger and are currently cancer free [2]. Other studies investigating the survivorship experience of young BCS have used a similar age cut-off [24]. The following eligibility criteria were used: participants were required to: have the ability to read and comprehend English and be over 18 years of age. They were excluded from the study if they were diagnosed with advanced stages of breast cancer (i.e., stage 4); experienced a breast cancer recurrence; had a previous history of other cancers; or had a previous history of chemotherapy or radiation for other cancers. We chose to apply these exclusion criteria to reduce variables that could confound our findings.

Participants were recruited through online advertisements on a variety of Canadian and American breast cancer support organization websites. The majority of the participants were recruited through the Avon Army of Women organization [25]. A total of 1113 English-speaking North-American young BCS were recruited. After removing both data corresponding to univariate and multivariate outliers $(\alpha=0.001)$ and excluding participants who did not fully complete the questionnaire, a total of 816 participants were included in our analyses ( $\mathrm{n}=559$ young BCS with children and $\mathrm{n}=257$ young BCS without children). Young BCS with children were then randomly divided into two groups via a randomizer program [26]: an exploratory sample of young BCS with children $(n=284)$, henceforth referred to as the EFA sample; and a confirmatory sample of young BCS with children $(\mathrm{n}=275)$. We also included a third group, a confirmatory sample of young BCS without children $(n=257)$, both confirmatory samples taken together, will henceforth be referred to as CFA samples. Our sample sizes were adequate for exploratory and confirmatory factor analyses and stable solutions and models [27]. 


\section{Procedure}

A link to an online survey, hosted by iSurvey.ca, was provided to prospective participants. An online consent form was integrated in the survey and participants were required to provide informed consent prior to continuing with the survey. The survey was approximately $45 \mathrm{~min}$ in length and it comprised of a variety of questionnaires addressing topics such as their experience of breast cancer survivorship (e.g., experience during diagnosis, treatment and remission) and psychosocial issues such as fear of recurrence and coping. Participants could interrupt the survey at any time and continue at a later time. If participants did not complete the survey in one sitting, they were sent an electronic message with a link to the survey. Two reminder messages were sent in total before the survey was marked as incomplete. The study was approved by the Research Ethics Board at the University of Ottawa. Due to the sensitive nature of the topic, participants who experienced distress were advised to contact their local distress center.

\section{Measures}

Demographic \& medical variables

A self-developed sociodemographic and medical questionnaire was administered. The sociodemographic questionnaire included questions that pertained to the participant's country of birth, ethnicity, age, relationship status, education and the age of their children. Participants were also asked to provide information about their medical history, such as their stage of diagnosis, type of surgery, adjuvant treatment and the presence of chronic noncancer health problems.

\section{The Medical Outcomes Study: short form (MOS-SF-36)}

The 36-item Medical Outcomes Study - short form (MOS-SF-36) [28] is a questionnaire that is used to record health-related quality of life in eight domains: physical functioning, social functioning, physical and emotional role limitations, mental health, vitality, pain and overall health perception. Scores range from 0 to 100 for each scale; higher scores are associated with better health and lower scores reflect substantial functional impairment, significant social and role limitations and poor health evaluations. The MOS-SF-36 has been shown to be a reliable and valid instrument across diagnoses, including cancer [28]. Cronbach's alphas for the exploratory and confirmatory samples in this study ranged from 0.80 to 0.94 , except for the General Health subscale; it had an alpha value of 0.44 .

\section{Perceived Stress Scale}

The Perceived Stress Scale (PSS) [29] is a ten-item measure that assesses participants' sense of control and manageability of events that have happened within the past month. Participants rate items using a five-point scale, ranging from 0 to 4 . Total scores range from 0 to 40, with higher scores signifying greater overall stress (an unpredictable, uncontrollable and overwhelming life). The PSS is a common measure used with breast cancer patients [19] and has good reliability and validity $(\alpha>0.86)$ [29]. For the current study, the PSS demonstrated strong reliability $(\alpha=0.92)$.

\section{Illness Intrusiveness Ratings Scale}

The Illness Intrusiveness Ratings Scale (IIRS) [30] is a 13-item questionnaire that measures the extent to which participants perceive their illness and/or its treatment as interfering with a variety of valued activities and interests. The IIRS comprises three subscales: instrumental (intrusiveness in health, work and finances); intimacy (intrusiveness in sexual life and relationship with one's partner); and relationship and personal development (intrusiveness in family and social relations, community involvement and religious expression). Participants answer each item on a seven-point scale, with higher scores reflecting greater illness intrusiveness. The IIRS has demonstrated good internal reliability $(\alpha>0.80)$ and validity in studies assessing a variety of chronic and health problems, such as breast cancer $[30,31]$. In this study, the exploratory and confirmatory analyses yielded Cronbach alphas of $0.81-0.91$.

\section{Concerns About Recurrence Scale}

The Concerns About Recurrence Scale (CARS) [14] is a 30-item questionnaire comprising five subscales, designed to assess one's fear of cancer recurrence. It first assesses one's overall fear via four questions on a six-point scale: frequency, consistency, potential for upset and intensity of fears. Overall fear refers to the fear of having a recurrence. The second section includes the remaining four subscales - health worries, womanhood worries, role worries and death worries - and examines the nature of the concerns using a four-point scale. Higher scores on each scale 
indicate higher fears. Cronbach's alphas for the exploratory and confirmatory samples in this study ranged from 0.88 to 0.94 , which is similar to its psychometric properties reported in other studies ( $\alpha=0.87-0.94)$ [14].

\section{Social Support Questionnaire}

The 40-item SSQ [32] assesses perceived emotional support from five different sources, including spouses, family members, friends, nurses and physicians using a five-point scale. The total scores range from 40 to 200; higher scores indicate greater levels of perceived support. Participants who indicated that they were not currently in a relationship did not complete the section on spousal support and their scores were adjusted accordingly. The SSQ has shown good reliability (Cronbach's alpha $=0.90$ ) and validity [32]. Cronbach's alphas for the exploratory and confirmatory samples in this study ranged from 0.92 to 0.93 .

\section{Factor analytic strategy for the current study}

To decipher patterns of common variance between the identified elements (18 elements) of well-being in young BCS with and without children, we followed a factor analysis sequence: exploratory factor analysis (EFA) on a first sample of 284 young BCS with children, confirmatory factor analysis (CFA) on a second sample of 275 young BCS with children and CFA invariance test for comparing the second sample of young BCS with children with young BCS without children $(\mathrm{n}=257)$.

EFA is a hypothesis-generating approach that assesses the covariance, or common variance, of the indicator variables, thereby generating a factor model; whereas CFA involves the testing of the predetermined factor structures [27]. In this study, we first performed an EFA with a group of BCS with children, followed by individual CFAs to test whether the generated model fits with a new sample of BCS with and without children. To ensure that our EFA and CFA samples were comparable in demographic and medical characteristics, we also performed group comparisons using analysis of variance and chi-square techniques.

Upon establishing a model that converged well for both groups, we used multiple-group CFA, also known as measurement of equivalence or invariance testing, to compare the factor structures between the groups [33]. A step-wise approach is required for testing the equivalence of a factor structure, starting with the least restricted model (configural model) and adding constraints to the model along the way [34]. In this study we will test, in this order, the invariance of indicators loading and intercept, as well as, invariance of factors variance, means and covariances. All analyses were performed using IBM SPSS version 23 and MPlus 7, in the case of the CFA (data available upon request).

\section{Results}

\section{Demographics \& medical characteristics}

Table 1 presents the demographic and medical characteristics of the samples. The results of the analysis of variance, and chi-square analyses on demographic differences, indicated that BCS without children in the CFA group were significantly younger and more likely to have achieved higher education and to be single than the other two groups. However, the average age difference was marginal ( 44 vs $46 \pm 7.7-9.2$ years). As for medical differences, BCS without children in the CFA sample tended to have less severe breast cancer, fewer mastectomies, more lumpectomies and were less likely to have received chemotherapy compared with both groups of mothers, although effect sizes were small (partial $\left.\eta^{2}=0.009-0.139\right)$.

\section{Exploratory factor analysis}

We performed an EFA on a sample of BCS with children to model the pattern of covariance among elements of well-being. Due to the nature of the variables, we were unable to meet the assumption of normality despite various transformation attempts. Therefore, we performed the EFA on untransformed data. As such, we used Principal Axis Factoring with promax rotation, which can be performed on data that are not normally distributed [35]. The initial analysis involved 18 variables, which consisted of all the subscales from the MOS-SF-36, IIRS, CARS and the total scores of the SSQ and PSS. We determined the number of factors to retain based on Kaiser's criterion of 1 and the inflexion in the scree plot. An indicator variable was considered to be associated with a factor if its loading was $\geq 0.30$ [36]. Through subsequent analyses, three subscales from the MOS-SF-36 (general health, role emotional and social functioning) were eliminated to obtain a parsimonious factor structure with few or no cross-loading items [36]. The final solution yielded a four-factor structure that accounted for $74 \%$ of the variance (see Table 2 for factor structure). 
Table 1. Group differences in demographic and medical variables for the exploratory factor and confirmatory factor

\section{analyses.}

Characteristic

\begin{tabular}{l} 
EFA $(n=284)$ \\
$M(S D)$ \\
$46(7.7)^{\star}$ \\
$16(9.3)$ \\
$39(4.3)$ \\
$7.3(6.8)$ \\
\hline
\end{tabular}

\begin{tabular}{ll} 
CFA 1 $(n=275)$ & CFA 2 $(n=257)$ \\
\hline$M(S D)$ & $M(S D)$ \\
$46(8.3)$ & $44(9.2)^{\star}$ \\
\hline $16(9.9)$ & N/A \\
\hline $39(4.5)$ & $38(5.9)$ \\
$6.9(7.0)$ & $6.3(5.9)$ \\
\hline
\end{tabular}

Current age

$27^{*} \quad 24 \quad 12$ *

- $\leq$ High school

10

- Associate/technical degree 10

- College/university degree 41

- Graduate school 22

Relationship status (\%)

- In a relationship $90^{*}$

- Not in a relationship $10^{*}$

$90^{*}$

\section{Working status (\%)}

\begin{tabular}{llll} 
- Working & 71 & 69 & 73 \\
\hline - Not working & 29 & 31 & 23
\end{tabular}

\section{Breast cancer stage (\%)}

$-0$

$-1 \quad 34$

$-11$

- III

\section{Surgery type (\%)}

- Unilateral mastectomy

- Bilateral mastectomy 29*

- Lumpectomy $35^{\star}$

$\begin{array}{lll}16 & 13 & 20^{\star} \\ 34 & 39 & 43 \\ 35 & 30 & 28 \\ 15 & 18^{\star} & 9^{\star}\end{array}$

\section{Chemotherapy (\%)}

\begin{tabular}{|c|c|c|c|}
\hline - Yes & 72 & $76^{*}$ & $66 *$ \\
\hline- No & 28 & $24 *$ & $34 *$ \\
\hline \multicolumn{4}{|c|}{ Radiation therapy (\%) } \\
\hline - Yes & 43 & 45 & 41 \\
\hline- No & 57 & 55 & 59 \\
\hline \multicolumn{4}{|c|}{ Hormone therapy (\%) } \\
\hline - Yes & 57 & 55 & 59 \\
\hline- No & 43 & 45 & 41 \\
\hline \multicolumn{4}{|c|}{ Other chronic health condition (\%) } \\
\hline - Yes & 23 & 26 & 25 \\
\hline- No & 77 & 74 & 75 \\
\hline
\end{tabular}

EFA analyses were completed with breast cancer survivors with children only, whereas CFA analyses were computed on breast cancer survivors with and without children (CFA 1 and CFA 2, respectively).

*Significant at $p<0.05$.

CFA: Confirmatory factor analysis; EFA: Exploratory factor analysis; M: Mean; N/A: Not applicable; SD: Standard deviation.

The first factor, recurrence worries, included worries related to health, role, death and womanhood $(0.99$, $0.86,0.78$ and 0.70 , respectively). It represented the extent to which women worried about the negative physical, psychological and social consequences of having a breast cancer recurrence. It is important to note that this factor did not measure worries about the possibility of having a breast cancer recurrence, but rather the content of these worries.

The second factor, physical health, included the ability to perform a variety of activities on a typical day, such as running and the extent of distance that one is able to walk (physical functioning: 0.91 ), the absence of limitations 
Table 2. Exploratory factor analysis pattern matrix and facture structure, including pattern loadings, communalities and the percentage of variance accounted for by each factor.

\begin{tabular}{|c|c|c|c|c|c|}
\hline Item & $F_{1}$ & $F_{2}$ & $F_{3}$ & $\mathrm{~F}_{4}$ & $h^{2}$ \\
\hline Health worries (CARS) & $0.99^{\dagger}$ & -0.06 & -0.04 & -0.10 & 0.997 \\
\hline Role worries (CARS) & $0.86^{\dagger}$ & -0.03 & 0.09 & 0.08 & 0.750 \\
\hline Death worries (CARS) & $0.78^{\dagger}$ & -0.04 & -0.04 & -0.07 & 0.603 \\
\hline Womanhood worries (CARS) & $0.70^{\dagger}$ & 0.16 & -0.04 & 0.18 & 0.529 \\
\hline Physical functioning (MOS-SF-36) & 0.03 & $0.91^{\dagger}$ & -0.06 & 0.14 & 0.641 \\
\hline Role physical (MOS-SF-36) & -0.02 & $0.79^{\dagger}$ & -0.03 & -0.10 & 0.718 \\
\hline Bodily pain (MOS-SF-36) & 0.00 & $0.74 \dagger$ & 0.12 & 0.11 & 0.551 \\
\hline Mental health (MOS-SF-36) & 0.02 & 0.00 & $0.85^{\dagger}$ & -0.08 & 0.812 \\
\hline Perceived stress (PSS) & -0.05 & 0.09 & $-0.75^{\dagger}$ & 0.21 & 0.703 \\
\hline Vitality (MOS-SF-36) & -0.04 & $0.39^{\dagger}, \ddagger$ & $0.64^{\dagger}$ & 0.06 & 0.758 \\
\hline Social support (SSQ) & -0.06 & -0.04 & $0.51^{\dagger}$ & 0.04 & 0.230 \\
\hline Intimacy (IIRS) & 0.02 & 0.01 & -0.09 & $0.66^{\dagger}$ & 0.521 \\
\hline Instrumental (IIRS) & -0.00 & $-0.49^{\dagger}, \ddagger$ & 0.18 & $0.66^{\dagger}$ & 0.881 \\
\hline Overall fears (CARS) & 0.06 & 0.22 & -0.13 & $0.65^{\dagger}$ & 0.390 \\
\hline Relationships/personal development (IIRS) & -0.04 & -0.23 & -0.08 & $0.57^{\dagger}$ & 0.610 \\
\hline Eigenvalue & 5.69 & 2.95 & 1.40 & 1.03 & - \\
\hline Percent of covariance & 37.94 & 19.70 & 9.35 & 6.86 & - \\
\hline \multicolumn{6}{|c|}{$\begin{array}{l}\text { Factor labels }-F_{1} \text { : Recurrence worries; } F_{2} \text { : Physical health; } F_{3} \text { : Psychological adjustment; } F_{4} \text { : Illness intrusiveness. } \\
\text { †Loadings over }|0.30| \text {. } \\
\ddagger \text { Identified crossloadings. }\end{array}$} \\
\hline
\end{tabular}

with work or other daily activities due to physical health (role physical: 0.79 ) and the absence of bodily pain and interference of normal activities due to pain (bodily pain: 0.74 ).

The third factor, psychological adjustment, included mental health (0.85), perceived stress $(-0.75)$, vitality $(0.64)$ and social support (0.51). The loading items on this factor suggest that psychological adjustment represents the extent to which women are free of anxiety, depression and stress, in other words, high levels of emotional wellbeing. The feeling of energy or liveliness (i.e., vitality) and the presence of social support, is also essential in positive psychological adjustment.

The last factor, illness intrusiveness, included measures of interference in intimate relationships and sexual life (intimacy: 0.66); interference in instrumental life domains such as work, health and finances (instrumental: 0.66); the extent to which women feared having a recurrence (overall fears: 0.65 ); and interference in community involvement, family and social relations and religious expression (relationships/personal development: 0.57 ). Higher levels of feelings of interference in any of the domains listed above or higher fears of recurrence contributed to greater extent of illness intrusiveness.

Finally, as shown in Table 2, we identified two indicator variables that cross-loaded on other factors - namely, vitality and instrumental, on physical health ( 0.39 and -0.49 , respectively). Although cross-loadings identified in an EFA can be removed from subsequent models in a step-wise manner [36], we chose to retain the two cross-loadings. We chose to retain vitality because studies in the well-being literature identify vitality as an important construct in assessing well-being, whilst acknowledging that it often cross-loads onto other factors [37,38]. Moreover, the current literature supports our finding that vitality may be related to physical health: it has been suggested that vitality measures the extent to which women felt 'tired and worn out', a state closely resembling fatigue which has been associated with worse physical well-being [39]. Therefore, removing vitality from the model would greatly affect our examination of well-being. We also chose to retain instrumental because it is one of the marker variables for the illness intrusiveness factor. We also reasoned that it is to be expected to cross-load on physical health because despite being a separate factor, one's physical health can affect one's feelings of illness intrusiveness $(r=-0.74 ; p<$ 0.001). Indeed, the MOS-SF-36 defines instrumental intrusiveness as interference in health, work and finances [28]. 


\begin{tabular}{|c|c|c|c|c|c|c|c|c|c|c|}
\hline Models & df & $\mathrm{SB} \chi^{2}$ & SRMSR & RMSEA & TLI & CFI & $\Delta \mathrm{CFI}$ & $\Delta \mathrm{df}$ & $\Delta \mathrm{SB} \chi^{2}$ & SC \\
\hline \multicolumn{11}{|l|}{ CFA solutions BCS with children $(n=275)$} \\
\hline 4-factor solution & 84 & 228.346 & 0.047 & 0.079 & 0.921 & 0.937 & - & - & - & 1.065 \\
\hline 4-factor with vitality $\mathrm{CL}$ & 83 & 194.222 & 0.043 & 0.070 & 0.938 & 0.951 & 0.014 & 1 & $43.823^{\star}$ & 1.068 \\
\hline $\begin{array}{l}\text { 4-factor with vitality and instrumental } \\
\mathrm{CL}\end{array}$ & 82 & 171.466 & 0.038 & 0.063 & 0.950 & 0.961 & 0.010 & 1 & $16.239^{\star}$ & 1.062 \\
\hline \multicolumn{11}{|c|}{ CFA solutions for BCS without children $(n=257)$} \\
\hline 4-factor solution & 84 & 276.979 & 0.059 & 0.095 & 0.876 & 0.901 & - & - & - & 1.003 \\
\hline 4-factor with vitality $\mathrm{CL}$ & 83 & 243.989 & 0.056 & 0.087 & 0.896 & 0.917 & 0.016 & 1 & $28.719^{\star}$ & 1.001 \\
\hline $\begin{array}{l}\text { 4-factor with vitality and instrumental } \\
\mathrm{CL}\end{array}$ & 82 & 212.187 & 0.052 & 0.079 & 0.914 & 0.933 & 0.024 & 1 & $27.693^{*}$ & 0.999 \\
\hline \multicolumn{11}{|l|}{ Invariance testing } \\
\hline Step 1: configural model & 164 & 382.424 & 0.045 & 0.071 & 0.934 & 0.948 & - & - & - & 1.031 \\
\hline Step 2: invariant loadings & 177 & 397.390 & 0.057 & 0.068 & 0.938 & 0.948 & 0.000 & 13 & 17.186 & 1.045 \\
\hline Step 3: invariant intercepts & 188 & 429.073 & 0.061 & 0.069 & 0.936 & 0.943 & 0.005 & 11 & $32.022^{*}$ & 1.042 \\
\hline $\begin{array}{l}\text { Step 3A: release of overall fears } \\
\text { intercept }\end{array}$ & 187 & 419.460 & 0.061 & 0.068 & 0.938 & 0.945 & 0.003 & 10 & $22.057^{\star}$ & 1.043 \\
\hline $\begin{array}{l}\text { Step 3B: release of social support } \\
\text { intercept }\end{array}$ & 186 & 413.460 & 0.060 & 0.068 & 0.939 & 0.946 & 0.002 & 9 & 15.908 & 1.043 \\
\hline Step 4: invariant factor variances & 190 & 416.854 & 0.060 & 0.067 & 0.941 & 0.946 & 0.000 & 4 & 2.542 & 1.040 \\
\hline Step 5: invariant factor means & 194 & 419.170 & 0.059 & 0.066 & 0.942 & 0.947 & 0.001 & 4 & 2.001 & 1.039 \\
\hline Step 6: invariant factor covariances & 200 & 445.914 & 0.068 & 0.068 & 0.939 & 0.942 & 0.005 & 6 & $25.571^{\star}$ & 1.042 \\
\hline Step 6A: release covariance II with PA & 199 & 426.048 & 0.064 & 0.065 & 0.943 & 0.946 & 0.001 & 5 & 7.150 & 1.041 \\
\hline
\end{tabular}

\section{Confirmatory factor analysis}

We elected to perform two separate CFAs to determine whether the four-factor structure derived from the EFA could be replicated in a second sample of young BCS with and a sample of BCS without children. Confirmatory factor analyses using maximum likelihood robust estimation (MLR) were conducted for each group, which corrected the standard error of parameters in the model. Based on the results of the EFA, our confirmatory model included four factors and 15 indicator variables. We allowed the free estimation of the following two indicators on a secondary factor, as demonstrated in the EFA: vitality and instrumental. Allowing the free estimation of these two indicator variables lead to an "unbiased estimates of factor correlations notwithstanding the loss in parsimony associated with these models" [40]. The first item loadings of each factor, that is, health worries, physical functioning, mental health and intimacy, were constrained to 1.0 for the purposes of factor scaling. The intercepts of the first indicator variables in each factor were fixed to 0.0 in order to estimate factor means. After evaluating the fit of the CFA model in both groups, we sought to investigate the measurement equivalence of the CFA models.

Several indices were used to assess the model's goodness of fit with the data: the comparative fit index (CFI) and the Tucker-Lewis index (values of 0.95 or higher represent a good fit) [41]; the root-mean-square error of approximation (values between 0.05 and 0.08 indicate a fair fit) [42]; and the standardized root-mean-square residual (values lower than 0.08 indicate acceptable fit). Since MLR was used for model estimation, Satorra-Bentler scaled statistics $\left(\mathrm{SB} \chi^{2}\right)$ were computed for model comparison purposes, which were then used for the comparisons of nested models $(\triangle \mathrm{SB} \times 2)$ [43,44], which is used for comparing models that are estimated with MLR. We also examined the CFI differences between the models, as recommended by Cheung and Rensvold [45], with change greater than 0.002 in CFI indicating a notable difference between the models [46]. See Table 3 for the detailed stepwise CFA results.

\section{CFA solutions for BCS with \& without children}

As shown in Table 3, we tested a sequence of CFA solutions for BCS with and without children. First, we tested the four-factor solution, then we added the vitality cross-loading, and finally, we evaluated the four-factor solution with vitality and instrumental cross-loadings. The original four-factor solution produced an adequately fitting 
model for BCS with and without children, $\mathrm{SB} \chi 2(84)=228.346$ and $\mathrm{SB} \chi 2(84)=276.979$, respectively. But, the sequential estimation of cross-loadings significantly increased the fit of the model, with CFI, Tucker-Lewis index, root-mean-square error of approximation and standardized root-mean-square residual values within acceptable standards (see Table 3 for $\Delta \mathrm{SB} \chi^{2}$ statistics).

\section{Multiple-group CFA invariance}

Upon obtaining a model that fit well for both groups, we compared the factor structure using a multiple-group CFA invariance test. A stepwise procedure was used which followed the recommendations by Brown [33], including a test of equal form (i.e., configural model); a test of the equality of factor loadings; a test of the equality of indicator intercepts; a test of the equality of factor variances; a test of the equality of factor means; and a test of the equality of factor covariances. Figure 1 illustrates the results of the final multiple-group CFA model. The measurement (steps $1-3)$ and structural invariance results (steps 4-6) are presented in Table 3.

\section{Measurement invariance}

As shown in Table 3, the configural model displayed good fit indices, indicating that the CFA model was fitting well with both groups when estimated simultaneously. We then tested whether the loadings were equivalent across both groups. Equivalent loadings were found between groups, as indicated by a change of 0.000 in CFI and the nonsignificant $\triangle \mathrm{SB} \times 2$. This finding suggests that the indicator variables did not differ in their relative contribution to their respective factors.

Finally, the equivalence of indicator intercept was assessed. The invariance procedure revealed a significant $\triangle \mathrm{SB} \times 2$. Modification indices (MI) were used to evaluate the source of non-equivalence $(\mathrm{MI}=9.315)$. The overall fear indicator, $\tau_{1}=13.395, \mathrm{SE}_{1}=0.344 ; \tau_{2}=12.848, \mathrm{SE}_{2}=0.33$, was not equivalent and therefore released from its equivalence constraint, resulting in a modified model that was tested in Step $3 \mathrm{~A}$. The $\Delta \mathrm{SB} \times 2$ of the modified model was significant, and modification indices now revealed a nonequivalence of social support ( $\mathrm{MI}=5.909)$. Therefore, we released the intercept constraint of social support, $\tau_{1}=148.898, \mathrm{SE}_{1}=1.27 ; \tau_{2}=144.603, \mathrm{SE}_{2}=1.523$, in the subsequent model (Step 3B). This newly modified model was not significantly different, thus revealing partial invariance of intercepts. It is worth noting that based on $95 \%$ confidence intervals, the difference between the intercepts were not significantly different from one another. The intercepts non-equivalence of overall fear and social support is probably due to the known sensitivity of the chi-squared test [27].

\section{Structural invariance}

As shown in Step 4 (Table 3), equivalent factor variances were found between the groups. We then tested for invariant factor means, and equivalence was found between the groups. We proceeded with the evaluation of equivalence in factor covariance. Results indicated that this model was significantly different. To find the source of nonequivalence, we examined the model's MI, and it was noted that the covariance between psychological adjustment and illness intrusiveness had the highest $\mathrm{MI}(\mathrm{MI}=19.01)$. As such, we released the equivalence constraint of that parameter and re-examined the fit of the model. The new model (Step 6A) was not significantly different thus revealing partial invariance of the covariances. This last step demonstrated that BCS with and without children differed in the extent to which the two factors, psychological adjustment and illness intrusiveness, were inversely related. The relationship between these two factors was significantly stronger in BCS with children $(r=-0.779,95 \%$ CI: -0.711 , $-0.848)$ compared with BCS without children $(r=-0.525,95 \%$ CI: $-0.423,-0.627)$.

\section{Discussion}

This exploratory study sheds light on the well-being of young BCS with children and the impact of having children compared with those without, through a factor analytic assessment of five correlates of well-being: health-related quality of life (via the MOS-SF-36) [28], fear of recurrence (via the CARS) [14], illness intrusiveness (via IIRS) [30], perceived stress (via PSS) [29] and availability of social support (via SSQ) [32]. Four main factors contributed to wellbeing in young BCS with children: recurrence worries (i.e., the content of these fears), physical health, psychological adjustment and illness intrusiveness (including the presence of fear of cancer recurrence). We found a stronger relationship between psychological adjustment and illness intrusiveness for young BCS with children, suggesting that the lack of psychological adjustment, or psychological distress, is closely related to illness intrusiveness for this group. Since the illness intrusiveness factor included a measure of fear of cancer recurrence, it was included in this relationship as well. Consequently, psychological distress, illness intrusiveness and fear of recurrence co-occurred 


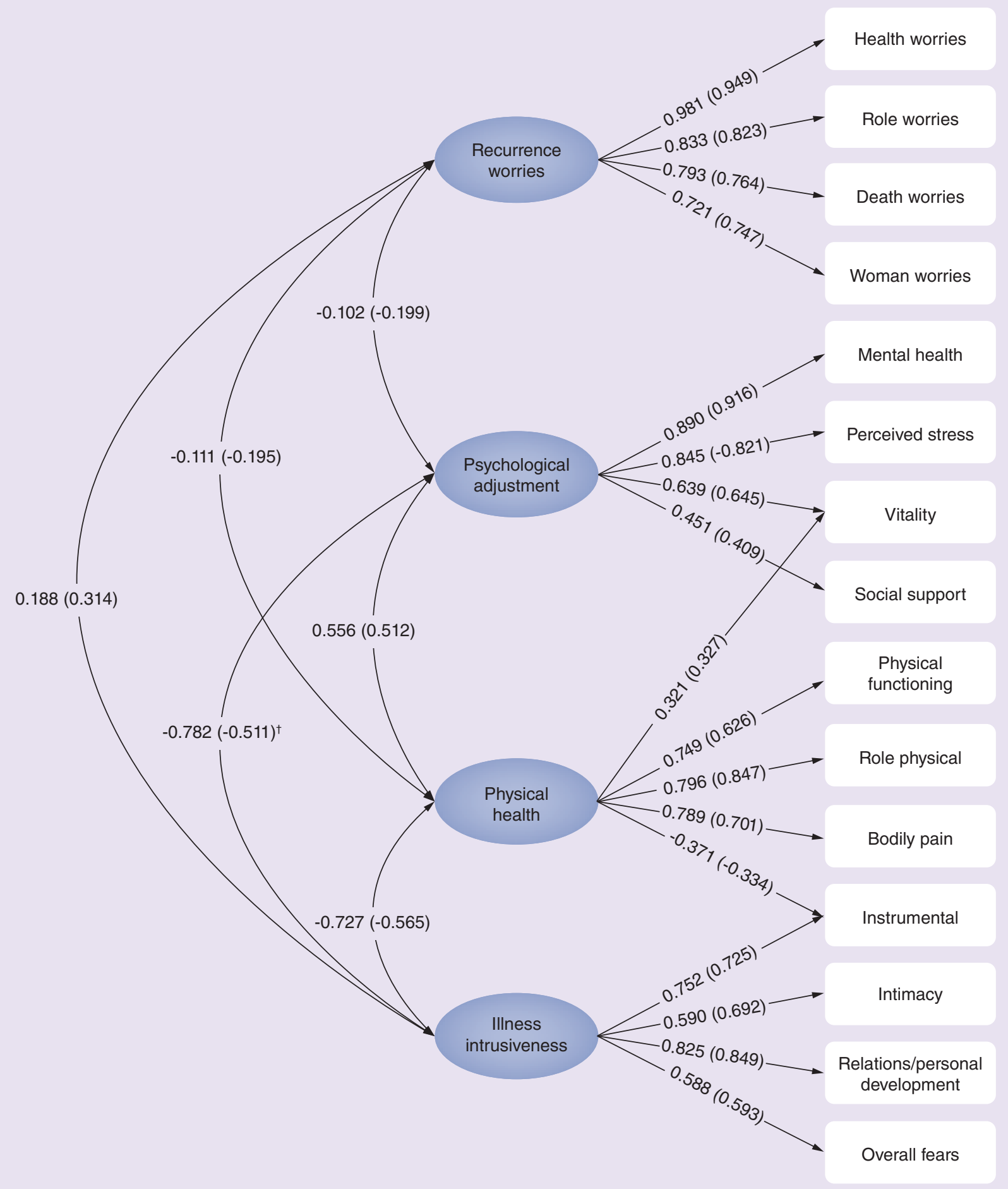

Figure 1. The multiple-group confirmatory factor analysis compares the factor structures between young breast cancer survivor with and without children ( $\mathrm{n}=\mathbf{2 7 5}$ and 257 , respectively).

Results for breast cancer survivor without children are displayed in brackets, and significant differences between groups are indicated $(\dagger)$. 
more frequently in young BCS with children than in BCS without children. This is the first study to demonstrate that having children while coping with breast cancer compromises their sense of well-being by exacerbating the relationship between psychological distress, illness intrusiveness and fear of recurrence.

The direction of the relationships among these three elements, and how they relate to parenting, is unclear and remains to be tested. It is possible that psychological distress in young BCS with children leads to greater illness intrusiveness and fear of cancer recurrence. Indeed, psychological distress has been shown to prospectively influence fear of cancer recurrence $[47,48]$; however, this may not be the case for illness intrusiveness. Although illness intrusiveness and psychological distress frequently co-occur [31], evidence suggests that illness intrusiveness exacerbates psychological distress and not the reverse [49].

It is also likely that illness intrusiveness and fear of cancer recurrence have the effect of heightening psychological distress in young BCS with children, possibly due to the potential inability to care for their children due to illness [5,7], thus supporting our finding that illness intrusiveness creates more psychological distress in this group. Elevated psychological distress could also be manifested as a result of the severity of their cancer diagnosis and the side effects and symptoms related to the course of treatment. Indeed, fatigue has been described as one of the most common and intrusive symptoms for young BCS with children, especially as it relates to meeting the demands of their illness as well as their children's needs for care $[5,8]$. Furthermore, fear of cancer recurrence in young BCS with children may be especially distressing. Previous research demonstrates that young BCS with children report greater fear of cancer recurrence because of the potential consequences a recurrence could have on their families, their ability to parent and their children's well-being [15,50]. Connell et al. [4] noted that the most commonly reported major personal concern in relation to fear of cancer progression and recurrence in a group of young BCS with children was the future welfare of their children. This research illustrates not only that fear of cancer recurrence is elevated among young BCS with children, but that these fears are also a source of significant concern and distress.

\section{Limitations}

Although our use of factor analysis precludes us from drawing firm causal interpretations, the aim of this exploratory study was to identify areas important to the well-being of BCS with children compared with survivors without children, using a parsimonious approach. Our overall sample, however, comprised women who were mainly Caucasian (over 93\%) with more than 25\% having completed graduate school, which could be due to online recruitment. However, we chose to recruit our participants online because it has been reported that roughly half of $\mathrm{BCS}$, and in particular younger women, obtain information and support primarily from the internet [51]. The relative infrequency of breast cancer in younger women made this method of recruitment ideal to reach this population. In addition, many review articles have concluded that participants in online studies were easily motivated to engage in research and produced results that were consistent with traditional research methods [52]. Our study will need to be replicated with more diverse samples to determine whether our results apply across demographic groups.

The demographics of our sample groups are related to higher socioeconomic status and ethnicity (Caucasian), but not medical variables such as breast cancer stage, or measures of psychological functioning [51]. The differences in demographic and medical characteristics were marginal and although some were statistically significant, the effect sizes of these differences were very small. This indicates that the differences were unlikely to have any practical implications in our results. Despite that, we acknowledge that the diversity of our sample is limited and may therefore not be representative of the population. We suggest readers to interpret our results with caution, especially for young BCS of other ethnic groups.

A further shortcoming is our definition of what constitutes 'young' BCS. Having a diagnosis under the age of 45 is not a universal age cut-off for a 'young' breast cancer diagnosis. But studies have shown that 45 is an appropriate age to choose because it is inclusive of young BCS with dependent children living at home [24].

\section{Future perspective: clinical \& research implications}

Parenting is an important concern for young BCS, yet little is known about the impact of having children on their sense of well-being. This exploratory study revealed that being a young BCS with children exacerbated the relationship between psychological distress, illness intrusiveness and fear of cancer recurrence, thus compromising their well-being. Our findings indicate that young BCS with children are most in need of screening and interventions in these areas, and should be incorporated in their medical care and visits. Nurses, for instance, should consider screening for illness intrusiveness and fear of cancer recurrence when they suspect psychological distress in a young BCS with children, in order to help reveal problematic areas in her recovery. 
Although there are existing validated tools to measure illness intrusiveness [49] and fear of cancer recurrence [53], these instruments have been mostly used in a research context. Their utility as short screening tools in a clinical context is an emerging area of study that can be implemented for screening young BCS with children [54]. Following the IIRS [49], for example, nurses can ask BCS " how much does your cancer and/or its treatment interfere with your relationship and social activities with your family?" Survivors who endorse some interference of their cancer with their family life could then be asked further questions about parenting concerns. This could clarify the nature of the difficulties and identify possible interventions such as normalization of concerns, psychoeducation or appropriate referrals to psychosocial resources (i.e., social work or psychology). Similarly, there are guidelines available that nurses can use to assess fear of recurrence [55]. From this assessment, BCS with children who endorse fear of cancer recurrence they consider frequent and bothersome would benefit from a referral to a psychosocial oncology specialist who could help them deal with elevated fear of cancer recurrence. At the present time, there is evidence that brief CBT interventions [56] can help survivors better manage their fear of cancer recurrence.

Lastly, there appears to be limited evidence of interventions designed specifically to address the unmet needs of young BCS, especially those with children. This is an important research gap that needs to be addressed. A 2016 review of psychosocial interventions for families with parental cancer (all types) [57] identified 19 different interventions and found that most were successful at reducing distress and improving quality of life, two of the important domains of functioning targeted by the present study. This review also assessed for barriers and facilitators for these psychosocial interventions. One of the main dimension of facilitators was the clinician's provision of psychosocial support services during the BCS' routine care, which can reduce the stigma associated with seeking mental health services and survivorship support. Nurses, who are arguably best positioned to systematically assess the unmet needs and desire for help of BCS with children, can play a key role in making support offers and/or advocating for provision of services that address the need of this vulnerable population.

\section{Conclusion}

Parenting and motherhood is an integral part of the lives of most individuals, and undoubtedly that includes those diagnosed with chronic illnesses. Paradoxically, little is known about the impact that having children may have on young BCS' sense of well-being and there are very few screening procedures and interventions designed for this vulnerable yet common group of individuals. To our knowledge, our exploratory study is the first one to compare the well-being factor structures of young BCS with and without children. Our results suggest that being a young BCS with children exacerbated the relationship between psychological distress, illness intrusiveness and fear of cancer recurrence, thus compromising their well-being. Our findings indicate that young BCS with children are most in need of screening and interventions in these areas and we urge the inclusion of validated screening tools in routine care and the early identification of BCS with children that may require additional survivorship care and support.

Financial \& competing interests disclosure

The authors have no relevant affiliations or financial involvement with any organization or entity with a financial interest in or financial conflict with the subject matter or materials discussed in the manuscript. This includes employment, consultancies, honoraria, stock ownership or options, expert testimony, grants or patents received or pending, or royalties.

No writing assistance was utilized in the production of this manuscript.

\section{Ethical conduct of research}

The authors state that they have obtained appropriate institutional review board approval or have followed the principles outlined in the Declaration of Helsinki for all human or animal experimental investigations. In addition, for investigations involving human subjects, informed consent has been obtained from the participants involved.

Open access

This work is licensed under the Creative Commons Attribution 4.0 License. To view a copy of this license, visit http://creativecomm ons.org/licenses/by/4.0/ 


\section{References}

Papers of special note have been highlighted as: $\bullet$ of interest

1. Weaver KE, Rowland JH, Alfano CM, McNeel TS. Parental cancer and the family. Cancer 116(18), 4395-4401 (2010).

2. Centers for Disease Control and Prevention. Breast cancer in young women. www.cdc.gov

3. Knobf MT. Clinical update: psychosocial responses in breast cancer survivors. Semin. Oncol. Nurs. 27(3), E1-E14 (2011).

4. Connell S, Patterson C, Newman B. Issues and concerns of young Australian women with breast cancer. Support. Care Cancer. 14(5), 419-426 (2006).

5. Semple CJ, McCance T. Parents' experience of cancer who have young children: a literature review. Cancer Nurs. 33(2), 110-118 (2010).

- Provides a comprehensive review regarding the existing studies on parental cancer and their experience of enduring cancer as well as taking care of their children. A variety of studies were identified, but the majority of it focuses on mothers with a breast cancer diagnosis.

6. Billhult A, Segesten K. Strength of motherhood: nonrecurrent breast cancer as experienced by mothers with dependent children. Scand. J. Caring Sci. 17(2), 122-128 (2003).

7. Stiffler D, Haase J, Hosei B, Barada B. Parenting experiences with adolescent daughters when mothers have breast cancer. Oncol. Nurs. Forum. 35(1), 113-120 (2008).

8. Fisher C, O'Connor M. 'Motherhood' in the context of living with breast cancer. Cancer Nurs. 35(2), 157-163 (2012).

9. Schmitt F, Piha J, Helenius $\mathrm{H}$ et al. Multinational study of cancer patients and their children: factors associated with family functioning. J. Clin. Oncol. Off. J. Am. Soc. Clin. Oncol. 26(36), 5877-5883 (2008).

10. Osborn T. The psychosocial impact of parental cancer on children and adolescents: a systematic review. Psychooncology 16(2), 101-126 (2007).

- Provides a review of the parental cancer literature with a focus on the experiences and psychosocial outcomes of the child or adolescent.

11. Maggard MA, O’Connell JB, Lane KE, Liu JH, Etzioni DA, Ko CY. Do young breast cancer patients have worse outcomes? J. Surg. Res. 113(1), 109-113 (2003).

12. Bloom JR, Stewart SL, Chang S, Banks PJ. Then and now: quality of life of young breast cancer survivors. Psychooncology 13(3), $147-160$ (2004).

13. Alfano CM, Rowland JH. Recovery issues in cancer survivorship: a new challenge for supportive care. Cancer J. 12(5), 432-443 (2006).

14. Vickberg SM. The Concerns About Recurrence Scale (CARS): a systematic measure of women's fears about the possibility of breast cancer recurrence. Ann. Behav. Med. Publ. Soc. Behav. Med. 25(1), 16-24 (2003).

15. Lebel S, Beattie S, Arès I, Bielajew C. Young and worried: age and fear of recurrence in breast cancer survivors. Health Psychol. $32(6), 695$ (2013).

- Investigated the relationship between age, motherhood and fear of recurrence in breast cancer survivors.

16. Wan C, Silverstein K, Arès I, Bielajew C. Predictors of fear of recurrence in breast cancer survivors. Cancer Stud. Ther. 1, 1-8 (2016).

- Categorizes the types of fears/worries that are experienced by breast cancer survivors, and identifies characteristics of women who may be at greater risk for chronic psychological distress.

17. Callihan EB, Gao D, Jindal S et al. Postpartum diagnosis demonstrates a high risk for metastasis and merits an expanded definition of pregnancy-associated breast cancer. Breast Cancer Res. Treat. 138(2), 549-559 (2013).

18. Azim HA, Metzger-Filho O, de Azambuja E et al. Pregnancy occurring during or following adjuvant trastuzumab in patients enrolled in the HERA trial (BIG 01-01). Breast Cancer Res. Treat. 133(1), 387-391 (2012).

19. Beatty L, Lee C, Wade TD. A prospective examination of perceived stress as a mediator of the relationship between life-events and QOL following breast cancer. Br. J. Health Psychol. 14(4), 789-804 (2009).

20. Bloom JR, Stewart SL, Johnston M, Banks P, Fobair P. Sources of support and the physical and mental well-being of young women with breast cancer. Soc. Sci. Med. 53(11), 1513-1524 (2001).

21. Ashida S, Palmquist AEL, Basen-Engquist K, Singletary SE, Koehly LM. Changes in female support network systems and adaptation after breast cancer diagnosis: differences between older and younger patients. Gerontologist 49(4), 549-559 (2009).

22. Lee MK, Park S, Lee ES et al. Social support and depressive mood 1 year after diagnosis of breast cancer compared with the general female population: a prospective cohort study. Support. Care Cancer. 19(9), 1379-1392 (2010).

23. Kroenke CH, Rosner B, Chen WY, Kawachi I, Colditz GA, Holmes MD. Functional impact of breast cancer by age at diagnosis. J. Clin. Oncol. 22(10), 1849-1856 (2004).

24. Adams E, McCann L, Armes J et al. The experiences, needs and concerns of younger women with breast cancer: a meta-ethnography. Psychooncology 20(8), 851-861 (2011).

25. Army of Women. www.armyofwomen.org

26. Research Randomizer. www.randomizer.org 
27. Kline RB. Principles and Practice of Structural Equation Modeling. Guilford Publications, NY, USA.

28. Ware JE, Kosinski M, Dewey JE, Gandek B. SF-36 health survey: manual and interpretation guide. Quality Metric Inc., Lincoln, RI, USA.

29. Cohen S, Kamarck T, Mermelstein R. A global measure of perceived stress. J. Health Soc. Behav. 24(4), 385-396 (1983).

30. Devins GM, Binik YM, Hutchinson TA, Hollomby DJ, Barré PE, Guttmann RD. The emotional impact of end-stage renal disease: importance of patients' perceptions of intrusiveness and control. Int. J. Psychiatry Med. 13(4), 327-343 (1984).

31. Bloom JR, Stewart SL, Johnston M, Banks P. Intrusiveness of illness and quality of life in young women with breast cancer. Psychooncology 7(2), 89-100 (1998).

32. Northouse LL. Social support in patients' and husbands' adjustment to breast cancer. Nurs. Res. 37(2), 91-95 (1988).

33. Brown TA. Confirmatory Factor Analysis for Applied Research. Guilford Publications, NY, USA.

34. Vandenberg RJ, Lance CE. A review and synthesis of the measurement invariance literature: suggestions, practices, and recommendations for organizational research. Organ. Res. Methods. 3(1), 4-70 (2000).

35. Winter JCF de, Dodou D. Factor recovery by principal axis factoring and maximum likelihood factor analysis as a function of factor pattern and sample size. J. Appl. Stat. 39(4), 695-710 (2012).

36. Osborne JW, Costello AB. Best practices in exploratory factor analysis: four recommendations for getting the most from your analysis. Pan-Pac. Manag. Rev. 12(2), 131-146 (2009).

37. Huta V, Ryan RM. Pursuing pleasure or virtue: the differential and overlapping well-being benefits of hedonic and eudaimonic motives. J. Happiness Stud. 11(6), 735-762 (2010).

38. Ryan RM, Huta V, Deci EL. Living well: a self-determination theory perspective on eudaimonia. In: The Exploration of Happiness. Fave AD (Ed.). Springer, The Netherlands, 117-139 (2013). http://link.springer.com/chapter/10.1007/978-94-007-5702-8_7

39. Arndt V, Stegmaier C, Ziegler H, Brenner H. A population-based study of the impact of specific symptoms on quality of life in women with breast cancer 1 year after diagnosis. Cancer. 107(10), 2496-2503 (2006).

40. Asparouhov T, Muthén B, Morin AJS. Bayesian structural equation modeling with cross-loadings and residual covariances: comments on Stromeyer et al. J. Manag. 41(6), 1561-1577 (2015).

41. Hu L, Bentler PM. Cutoff criteria for fit indexes in covariance structure analysis: conventional criteria versus new alternatives. Struct. Equ. Model. Multidiscip. J. 6(1), 1-55 (1999).

42. Bollen KA, Long JS. Testing Structural Equation Models. SAGE, CA, USA.

43. Satorra A, Bentler PM. A scaled difference chi-square test statistic for moment structure analysis. Psychometrika. 66(4), 507-514 (2001).

44. Mplus. Chi-square difference testing using the Satorra-Bentler scaled chi square (2012). www.statmodel.com/chidiff.shtml

45. Cheung GW, Rensvold RB. Testing factorial invariance across groups: a reconceptualization and proposed new method. J. Manag. 25(1), $1-27$ (1999).

46. Meade AW, Johnson EC, Braddy PW. Power and sensitivity of alternative fit indices in tests of measurement invariance. J. Appl. Psychol. 93(3), 568-592 (2008).

47. Lebel S, Rosberger Z, Edgar L, Devins GM. Emotional distress impacts fear of the future among breast cancer survivors not the reverse. J. Cancer Surviv. 3(2), 117-127 (2009).

48. Liu Y, Pérez M, Schootman M, Aft RL, Gillanders WE, Jeffe DB. Correlates of fear of cancer recurrence in women with ductal carcinoma in situ and early invasive breast cancer. Breast Cancer Res. Treat. 130(1), 165 (2011).

49. Devins GM. Using the illness intrusiveness ratings scale to understand health-related quality of life in chronic disease. J. Psychosom. Res. 68(6), 591-602 (2010).

50. Melchior H, Büscher C, Thorenz A, Grochocka A, Koch U, Watzke B. Self-efficacy and fear of cancer progression during the year following diagnosis of breast cancer. Psychooncology 22(1), 39-45 (2013).

51. Fogel J, Albert SM, Schnabel F, Ditkoff BA, Neugut AI. Internet use and social support in women with breast cancer. Health Psychol. 21(4), 398-404 (2002).

52. Reips U-D. Standards for Internet-based experimenting. Exp. Psychol. 49(4), 243-256 (2002).

53. Thewes B, Butow P, Zachariae R, Christensen S, Simard S, Gotay C. Fear of cancer recurrence: a systematic literature review of self-report measures. Psychooncology 21(6), 571-587 (2012).

54. Lebel S, Ozakinci G, Humphris G et al. Current state and future prospects of research on fear of cancer recurrence. Psychooncology (2016). http://insights.ovid.com/pscyho-oncology/ponc/9000/00/000/current-state-future-prospects-research-fear/99521/014455499

55. Cancer Australia. Recommendations for the identification and management of fear of cancer recurrence in adult cancer survivors (2014). https://guidelines.canceraustralia.gov.au/guidelines/fear_of_recurrence/ch01.php

56. Butow PN, Bell ML, Smith AB et al. Conquer fear: protocol of a randomised controlled trial of a psychological intervention to reduce fear of cancer recurrence. BMC Cancer 13, 201 (2013). 
57. Inhestern L, Haller A-C, Wlodarczyk O, Bergelt C. Psychosocial interventions for families with parental cancer and barriers and facilitators to implementation and use - a systematic review - ProQuest. PLoS ONE 11(6), e0156967-e0156987 (2016). 
